\title{
Membrane-MEDYAN: Simulating Deformable Vesicles Containing Complex Cytoskeletal Networks Supplemental Information
}

\author{
Haoran $\mathrm{Ni}^{1}$ and ${ }^{*}$ Garegin A. Papoian (gpapoian@umd.edu) $)^{1,2,3}$ \\ ${ }^{1}$ Biophysics Program, University of Maryland, College Park, MD 20742 \\ ${ }^{2}$ Department of Chemistry and Biochemistry, University of Maryland, College Park, \\ MD 20742 USA \\ ${ }^{3}$ Institute for Physical Science and Technology, University of Maryland, College \\ Park, MD 20742 USA
}

August 28, 2021

\section{Simulation parameters and initial setups}

Table S1 lists the default parameters used for simulation for this work. Unlisted parameters used in cytoskeleton simulation, unless otherwise stated, take the same value as in. ${ }^{1}$ The membrane elastic parameters are chosen based on a prior study. ${ }^{[2]}$

Table S1: The parameters used in simulation.

\begin{tabular}{lll}
\hline Description & Symbol & Value \\
\hline Membrane tension & $\gamma$ & $0.02 \mathrm{pN} \mathrm{nm}^{-1}$ \\
Membrane bending rigidity & $\kappa$ & $100 \mathrm{pN} \mathrm{nm}$ \\
Membrane spontaneous curvature & $H_{0}$ & 0 \\
Membrane area elasticity & $k_{\text {area }}$ & $400 \mathrm{pN} \mathrm{nm}^{-1}$ \\
Membrane-filament bead repulsion constant & $k_{\text {rep }}$ & $1300 \mathrm{pNnm}^{3}$ \\
\hline
\end{tabular}

\subsection{Simulations for interactions between actin and membrane vesicle}

A simulation is set up for a vesicle system of radius $0.8 \mu \mathrm{m}$ with the parameters in Table $\mathrm{S} 2$ The seeding actin filaments are 81 linear filaments with 80 monomers each, and they are initialized in two different ways. In the first case, the seeding filaments are initialized within the vesicle with random position and orientation. In the second case, the seeding filaments are initialized as a bundle at the center of the vesicle, with the barbed ends pointing at two poles of the vesicle (with 41 pointing to one pole and 40 pointing to the other pole), and with the pointed ends lying on a plane at the center. The membrane surface has constant surface tension, and the bending and osmotic potentials are included.

Table S2: Parameters for vesicle bundle deformation simulation.

\begin{tabular}{ll}
\hline Description & Value \\
\hline Actin monomer concentration & $0.1 \mathrm{~mol} / \mathrm{m}^{3}$ \\
$\alpha$-actinin cross-linker concentration & $5 \times 10^{-3} \mathrm{~mol} / \mathrm{m}^{3}$ \\
Myosin motor concentration & $5 \times 10^{-5} \mathrm{~mol} / \mathrm{m}^{3}$ \\
\hline
\end{tabular}




\subsection{Perpendicular bundle protrusion against the membrane}

The simulation starts with a planar sheet of membrane of size $1 \mu \mathrm{m} \times 1 \mu \mathrm{m}$, with boundary vertices fixed and a constant surface tension of $0.02 \mathrm{pN} \mathrm{nm}^{-1}$. The bundled filaments are initially perpendicular to the surface of the membrane, each with length $432 \mathrm{~nm}$, and the filaments are separated by a nearest distance of $40 \mathrm{~nm}$. The barbed ends of the actin filaments point towards the membrane, and their the initial distances from the barbed ends to the membrane are $48 \mathrm{~nm}$. We denote the direction of barbed end polymerization as the $+z$ direction. The segments at the pointed ends of the actin filaments are fixed in the space to provide support.

\subsection{Non-perpendicular bundle protrusion against the membrane}

The simulations are initiated with a bundle of 7 filaments spaced $45 \mathrm{~nm}$ apart. The initial lengths of the filaments are $432 \mathrm{~nm}$ and the angle $\alpha$ between the direction of the filament and the normal vector of the membrane varies. The concentration of the G-Actin monomer is fixed, and the simulation parameters are listed in Table $\mathrm{S} 3$.

Table S3: Parameters for filament bundle polymerization with different contact angles with the membrane

\begin{tabular}{ll}
\hline Description & Value \\
\hline G-Actin monomer concentration & $0.1 \mathrm{~mol} / \mathrm{m}^{3}$ \\
-actinin cross-linker concentration & $2 \times 10^{-3} \mathrm{~mol} / \mathrm{m}^{3}$ \\
Reference membrane tension $\left(\gamma_{0}\right)$ & $0.02 \mathrm{pN} \mathrm{nm}{ }^{-1}$ \\
Reference membrane bending rigidity $\left(\kappa_{0}\right)$ & $100 \mathrm{pN} \mathrm{nm}$ \\
\hline
\end{tabular}

\section{Representation of membrane surface using a triangular mesh}

We discretize the 2-dimensional surface $\mathcal{M}$ into a triangulated meshwork $\hat{\mathcal{M}}$. The elements in the mesh include sets of vertices $V$, edges $E$ and triangles $T$, which are described in the main text. The 1-ring neighbor vertices $N_{v, 1}$ and the 1-ring neighbor triangles $N_{t, 1}$ are also defined in the main text. Two triangles $t_{i}, t_{j}$ are called adjacent if $t_{i} \cap t_{j} \in E$. Now, given a vertex $v \in V$, if we define a graph with the nodes being elements in $N_{t, 1}(v)$, and two nodes are connected if and only if two triangles share the vertex $v$ and are adjacent, then this graph can be divided into a set of connected components, and for each connected component, the collection of nodes (triangles) is called a fan around the vertex $v$.

To enforce a manifold-like structure, more rules need to be enforced on the elements of $\hat{\mathcal{M}}$ : (1) Every vertex must exist in at least 1 triangle. This also implies that every vertex exists in at least 2 edges. (2) Every edge must show up in no more than 2 triangles. By definition, an edge shows up in at least 1 triangle. We call the edge that shows up in exactly 1 triangle the border edge. (3) For any $v \in V$, there is exactly one fan around $v$. Here are some intuitions behind the rules. Rule (1) is to make sure that there is no vertex that is not "connected to" another vertex. Rule (2) prevents more than 2 triangles sharing the same edge, which leads to a non-manifold structure. Rule (3) is to ensure that each vertex exists at exactly one location on the surface $\hat{\mathcal{M}}$. Without it, Multiple locations on $\hat{\mathcal{M}}$ can share one vertex. See Fig. S1

If the original manifold $\mathcal{M}$ is orientable, $\hat{\mathcal{M}}$ can also be defined with an orientation. All the element definitions are the same with the previous definitions except for the following notable changes. A triangle $t \in T$, instead of being a set of 3 vertices, is now defined as an equivalence class identified by 3 distinct vertices $\left[v_{i}, v_{j}, v_{k}\right]$, with the equivalence being the cyclic permutation of index $i, j, k$. In other words, $\left[v_{i}, v_{j}, v_{k}\right]=\left[v_{j}, v_{k}, v_{i}\right]=\left[v_{k}, v_{i}, v_{j}\right]$. However, we still mandate that the set of vertices in each triangle is distinct. For example, although $\left[v_{1}, v_{2}, v_{3}\right] \neq\left[v_{1}, v_{3}, v_{2}\right]$, at most one of the two 
(a)

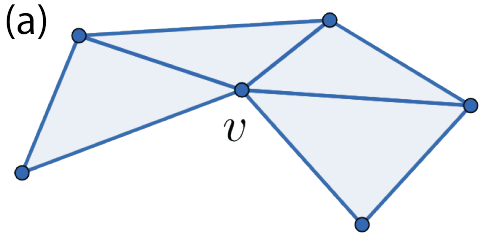

(c)

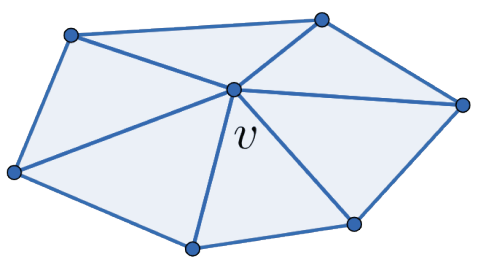

(b)

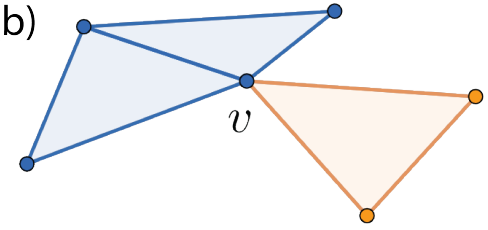

(d)

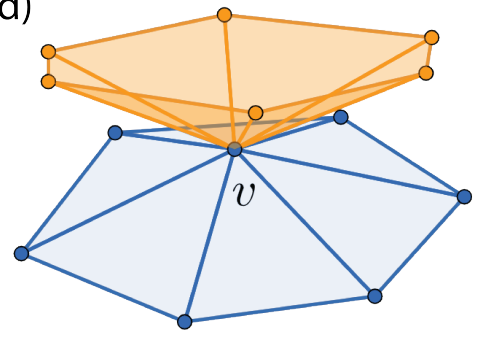

Figure S1: Examples of fans around the vertex $v$. Multiple fans are labeled using different colors. The configurations (a) and (c) each has one fan. The configurations (b) and (d) each has two fans, which is forbidden in the mesh.

is allowed to show up in $T$. In other words, the triangle can still be viewed as a set of 3 vertices, but with the additional information of the order of the vertices. Then, an edge is defined similarly as before (treating each triangle as a set of 3 vertices), but now it is also useful to define a set of directed edges (or half edges) $H$, each element being an ordered pair of distinct vertices $\left(v_{i}, v_{j}\right)$, so $H=\left\{\left(v_{i}, v_{j}\right) \in V \times V:\left\{v_{i}, v_{j}\right\} \in E\right\}$. Apparently, for each edge $\left\{v_{i}, v_{j}\right\}$, there are 2 corresponding half edges $\left(v_{i}, v_{j}\right),\left(v_{j}, v_{i}\right)$. Now the rules to enforce a manifold-like structure applies as well, but another rule needs to be added to account for the orientation. A half edge $h=\left(v_{i}, v_{j}\right)$ is associated with a triangle $t$ if $t=\left[v_{i}, v_{j}, v_{k}\right]$ for some $v_{k}$. Rule (4): For any edge that shows up in 2 triangles, each of the half edge corresponding to the edge must be associated with exactly one of the triangles. The intuition for this rule is that, if two oriented triangles are adjacent, the oriented normal vectors poke out from the same side of the surface.

In this work, we use oriented triangular mesh to represent an orientable membrane surface. If a non-orientable surface is required, one can extend the existing orientable mesh to an oriented double cover of the non-orientable mesh, where the set of vertices in each triangle being distinct is no longer required.

\section{Benchmark on membrane adaptive remeshing}

See Fig. S2 for the result of a benchmark of our adaptive remeshing algorithm.

\section{Membrane bending energy on discretized surface}

The bending terms in the Helfrich Hamiltonian can be used for computing the bending energy of the membrane $\mathcal{M}^{3 / 4}$

$$
E_{\text {bending }}=\int_{\mathcal{M}} d S\left(2 \kappa_{H}\left(H-H_{0}\right)^{2}+\kappa_{G} K\right)
$$

where $H$ is the mean curvature, $K$ is the Gaussian curvature, $H_{0}$ is the spontaneous curvature, $\kappa_{H}$ and $\kappa_{G}$ are the bending modulus and the saddle splay modulus respectively. Note that here the mean curvature $H$ stands for "the mean of two principal curvatures", while some other literatures 
(a)

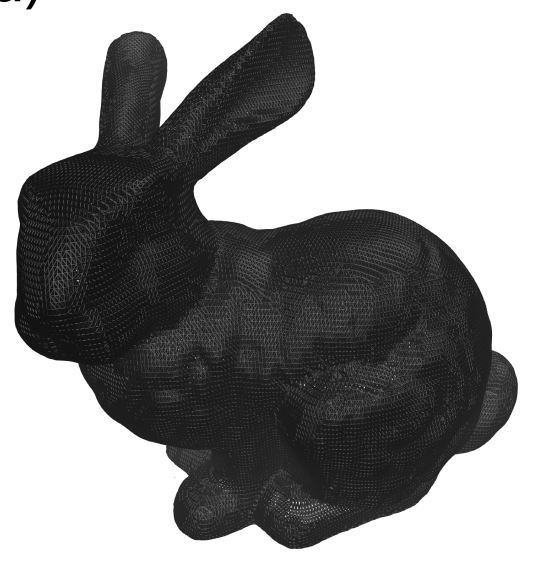

(b)

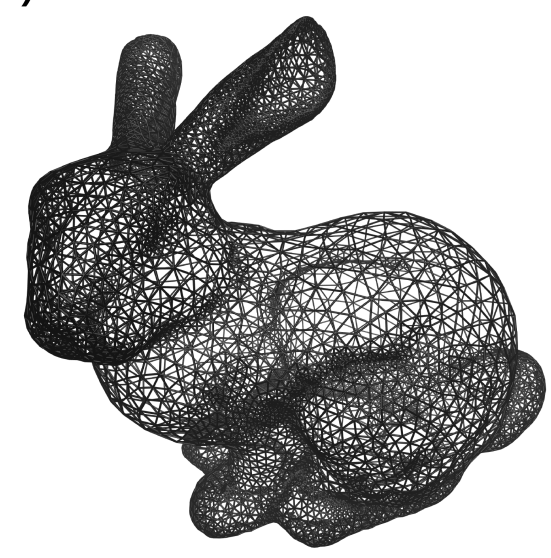

Figure S2: The remeshing algorithm generates anisotropic mesh with adaptable sizes suitable for simulation. Left: before remeshing; Right: after remeshing. (a) Showing the reconstructed mesh of the bunny model from the Stanford Computer Graphics Laboratory. The mesh is very dense and largely anisotropic. (b) The bunny mesh after the remeshing. The vertices are sparser than the original mesh, especially in relatively flat regions. Additionally, the vertex density increases in regions with higher local curvature.

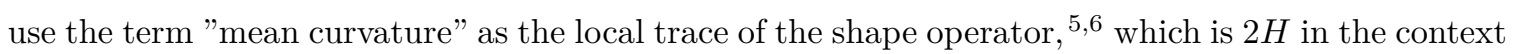
of this article.

For a membrane with fixed topology and boundary conditions, the contribution from the Gaussian curvature is a constant, so for most of our purposes we do not care about the second term in Eq. 1.

\subsection{Edge based curvature and bending energy estimation}

When a smooth surface is represented by the discretized triangles, the curvature on the surface can be viewed as being concentrated on the edges. 6 Consequently, a natural way of computing the discrete surface bending energy is to use the dihedral angle formula. $\frac{517}{79}$

$$
\hat{E}_{\text {bending }}=\tilde{\kappa} \sum_{\alpha, \beta \in T}\left(1-\left\langle\boldsymbol{n}_{\alpha}, \boldsymbol{n}_{\beta}\right\rangle\right)
$$

where $\tilde{\kappa}$ is the force constant, $T$ is the set of all the triangles in the mesh, and $\alpha$ and $\beta$ are adjacent oriented triangles with unit normal $\boldsymbol{n}_{\alpha}$ and $\boldsymbol{n}_{\beta}$. It is one of the simplest ways to implement the bending energy, but the relation between the constant $\tilde{\kappa}$ and the real physical constants should be carefully considered.

It is shown $n^{5}$ that under the continuum limit, Eq. 2 is equivalent to

$$
E_{\text {bending }}=\kappa \int_{\mathcal{M}} d S\left(2 H^{2}-K\right)
$$

which is the Helfrich energy when $H_{0}=0, \kappa_{H}=\kappa$ and $\kappa_{G}=-\kappa$. However, the relationship between $\kappa$ and $\tilde{\kappa}$ depends on the overall shape of the membrane, $\frac{10}{10}$ so for a membrane with arbitrary shape, this method might not converge to the Helfrich bending energy even at continuum limit. One of the origins of the difficulty is that while the discretized integral of mean curvature converges in the continuum limit, the discretized integral of squared mean curvature is singular. $\frac{10 \mid 11}{}$ The integral of 
mean curvature is still useful, however, when the spontaneous curvature $H_{0}$ is not zero, and then a linear integral of mean curvature can be extracted from the Helfrich free energy and estimated based on dihedral angle between edges

$$
\begin{aligned}
E_{\text {bending,linear }} & =-4 \kappa_{H} H_{0} \int_{\mathcal{M}} d S H \\
& \approx-4 \kappa_{H} H_{0} \sum_{\alpha, \beta \in T} l_{\alpha \beta} \arccos \left(\left\langle\boldsymbol{n}_{\alpha}, \boldsymbol{n}_{\beta}\right\rangle\right)
\end{aligned}
$$

where $l_{\alpha \beta}$ is the length of the edge shared by neighboring triangles $\alpha$ and $\beta$, and the approximation comes from the discretization.

\subsection{Vertex based curvature and bending energy estimation}

Despite the singularity of integral of squared mean curvature on a discretized triangular surface, one can still estimate the local mean curvature value around each vertex and use that to compute the bending energy. 11

$$
E_{\text {bending }}=2 \kappa_{H} \int_{\mathcal{M}} d S\left(H-H_{0}\right)^{2} \approx 2 \kappa_{H} \sum_{v \in V} \hat{A}(v)\left(\hat{H}(v)-H_{0}(v)\right)^{2}
$$

where $V$ is the set of all vertices, and $\hat{A}(v)$ and $\hat{H}(v)$ are the area and the mean curvature estimated around vertex $v$ respectively.

Many ways of estimating $\hat{A}(v)$ and $\hat{H}(v)$ exist. ${ }^{6]}$ Currently, in MEDYAN, the area around the vertex is a third of the sum of all the neighboring triangles sharing the vertex, and the curvature is estimated using the following formula 11 12

$$
\hat{H}\left(v_{i}\right)=\frac{\left\langle\nabla_{i} A, \nabla_{i} V\right\rangle}{2\left\langle\nabla_{i} V, \nabla_{i} V\right\rangle}
$$

where $A$ is the total area of the membrane, and $V$ is the total volume inside the membrane, which allows for continuous signed local curvature (signedness for curvature is important when $H_{0} \neq 0$ in Eq. 5), and avoids numerical singularity when $\nabla_{i} A=0$ on flat surfaces. In the continuum limit, $\nabla A \| \nabla V$ and are both along the normal direction, but numerically in a discretized mesh, there can be misalignments between the vectors mentioned above, and under extreme conditions these two vectors can even try to become perpendicular and produce unphysical geometries. A method with better numerical stability is by directly estimating the unsigned version of $\hat{H}\left(v_{i}\right)$

$$
\hat{H}\left(v_{i}\right)=\frac{\left\|\nabla_{i} A\right\|}{2\left\|\nabla_{i} V\right\|}
$$

This provides better numerical stability against several unphysical geometries in the first implementation. But in this case, we cannot directly incorporate the spontaneous curvature because the orientation information is lost in the equation. Nevertheless, the extra linear term can be extracted and computed in Eq. 4 .

\subsection{Comparing curvature estimation algorithms}

We tested various methods to compute local mean curvatures using the red blood cell (RBC) example of Guckenberger et al. $14[15$ The RBC is bi-concave and axisymmetric, and is specified by the following equation

$$
z= \pm \frac{R}{2} \sqrt{1-r^{2}}\left(C_{0}+C_{1} r^{2}+C_{2} r^{4}\right)
$$

where $r:=\sqrt{x^{2}+y^{2}} / R$ and $C_{0}=0.2072, C_{1}=2.0026$ and $C_{2}=-1.1228 .1416$ We applied the following setups as described in the article: $\frac{14}{14}(1) R=1$, making the curvature dimensionless; (2) The polar angle $\theta:=\operatorname{arccot}(z / r) ;(3)$ The maximum analytical mean curvature is $\left|H_{\max }\right| \approx$ 
(a)

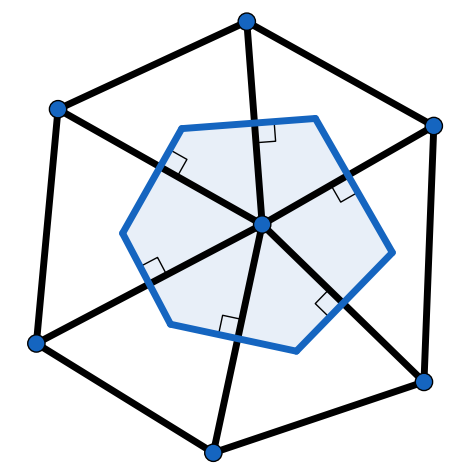

(c)

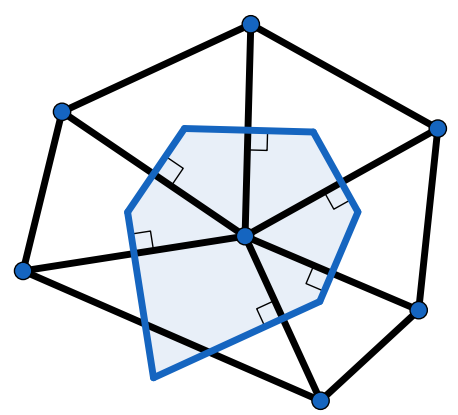

(b)

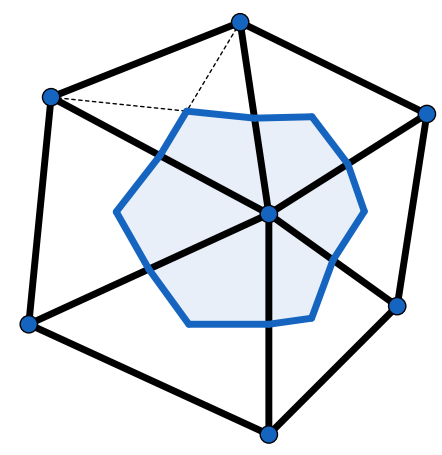

(d)

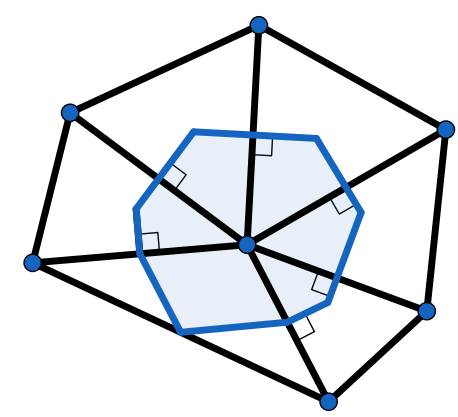

Figure S3: Ways of estimating the area at a vertex. The area is estimated from the region that is marked with blue shade. (a) Voronoi cell of the center vertex. (b) $1 / 3$ of sum of area of all neighboring triangles around the center vertex. (c) Voronoi cell of the center vertex when obtuse triangles are involved. Therefore, area estimation using Voronoi cell could suffer from great numerical errors when bad-quality triangles are involved. (d) Mixed area of the center vertex when obtuse triangles are involved. 13 
2.20098, which will be used as the denominator when calculating the relative error in curvature estimate, because the mean curvature can be zero at some points where the relative error is not well defined.

We tested 4 different ways of estimating the local curvature. Method A: The signed curvature estimate in Eq. 6. Method B: The unsigned curvature estimate in Eq. 7. Method C: Curvature estimate by applying the discretized Laplace-Beltrami operator, described by Eq. 8 by Meyer et al,, 13 but using $A_{\text {Voronoi }}$ instead of $A_{\text {mixed }}$ in the denominator; Method D: Similar to Method C, but exactly described by Eq. 8 by Meyer et al, 13 using $A_{\text {mixed }}$ to avoid pathological Voronoi areas in obtuse triangles. Out of these 4 methods, without relying on the normal vector at each vertex, only Method A naturally gives a signed mean curvature, while the rest gives unsigned mean curvatures.

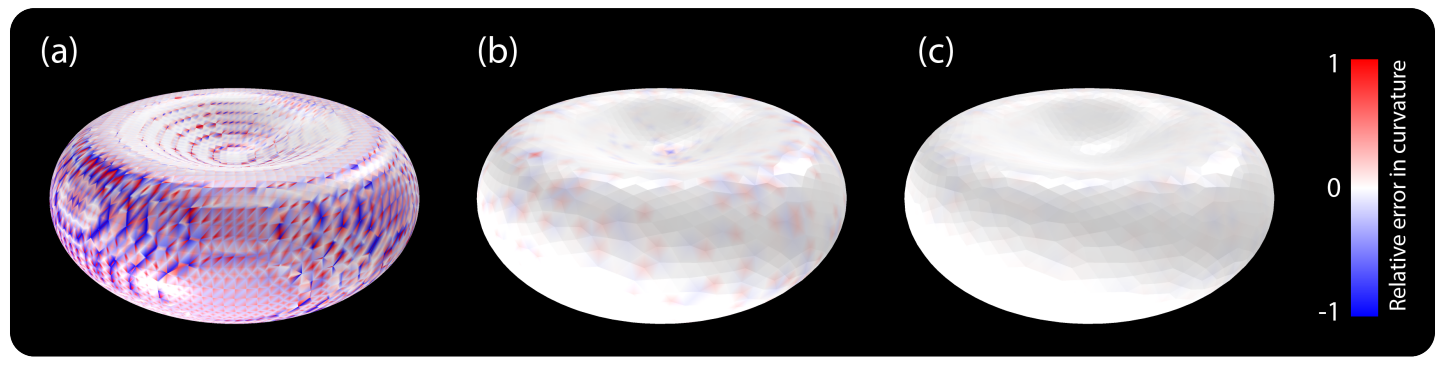

(d)

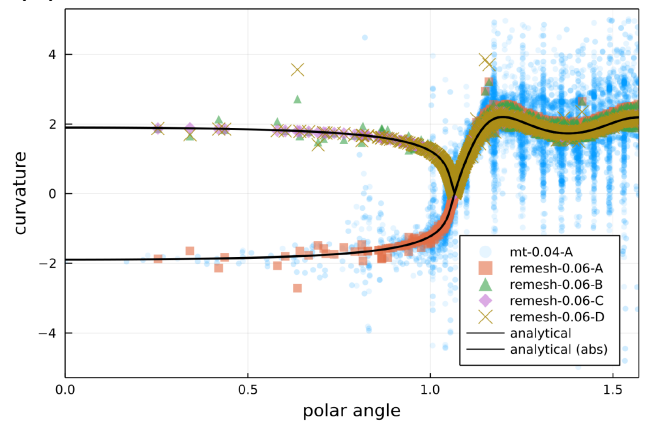

(e)

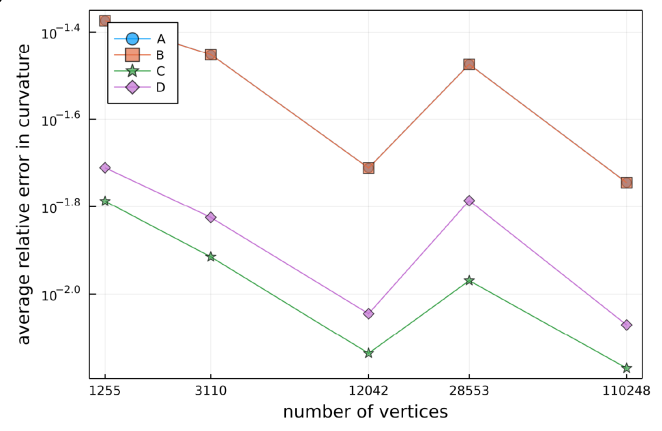

Figure S4: Result of curvature estimate benchmark on a red blood cell (RBC). (a) The relative error of curvature estimate using method A on the mesh generated by marching tetrahedra algorithm with a box size of 0.04. (b) The relative error of curvature estimate using method A on the mesh adapted by the remeshing algorithm (maximum recommended edge length: 0.06). (c) The relative error of curvature estimate using method $\mathrm{C}$ on the mesh adapted by the remeshing algorithm (maximum recommended edge length: 0.06). (d) The curvature profile of the upper half of the RBC, characterized by the polar angle of the vertices. (e) The averaged relative errors in curvature as a function of number of vertices in the mesh. The increasing number of vertices in the mesh is obtained by the remeshing algorithm, with maximum recommended edge length being 0.1, 0.06, 0.03, 0.02 and 0.01, respectively. The error data for Method A and B overlap on top of each other.

We initialized the RBC surface using the marching tetrahedra algorithm, $\frac{17}{17}$ with box size 0.04 . The surface is optimized by moving the generated vertices as close to the analytical surface as possible, using gradient descent algorithm. The curvature profile is recorded using Method A. Then the system is remeshed, with maximum recommended edge length ranging in $\{0.1,0.06,0.03,0.02,0.01\}$, leading to increasingly fine-grained meshes. The surface remeshing is coupled with the gradient descent optimization to make sure that the vertices accurately lie on the analytical RBC surface. For each number of vertices the curvature profile is recorded using all 4 methods described above. The results are displayed in Fig. S4 For RBC shapes, Method A and B provide good enough 
(average error is about 3\%) and comparable accuracy across various mesh granularities (Fig. S4b/e). Meyer's methods (Method C and D) provide better accuracy of $1.5 \%$ for most of the cases (Fig. $\mathrm{S} 4 \mathrm{f} / \mathrm{e}$ ). We would like to note that the initial mesh generated by the marching tetrahedra algorithm results in notably higher curvature errors (using Method A), indicating the importance of remeshing (Fig. S4a/b/d). We used Method A and B in our final model despite their slightly higher errors than Methods C and D, because they provide better numerical stability for extreme cases where the surface undergoes large deformations.

\section{Derivation of triangle-bead volume exclusion interaction}

The triangle-bead volume exclusion interaction takes the following form:

$$
\hat{E}_{\mathrm{excl}}=k_{\mathrm{excl}} \int_{\hat{\mathcal{M}}} d S \frac{1}{d^{n}}=k_{\mathrm{excl}} \sum_{t \in T} \int_{t} d S \frac{1}{d^{n}}
$$

Here we derive the explicit analytical result when $n=4$ and will discuss the geometric interpretation of some terms in the result.

\subsection{Evaluating the integral}

The integration can be done as follows. Suppose we have a triangle $t$, with 3 vertices $v_{0}, v_{1}, v_{2}$ with Cartesian coordinates $x_{0}, x_{1}, x_{2}$. Let $r_{01}=x_{1}-x_{0}$ and $r_{12}=x_{2}-x_{1}$, any point $q$ on the triangle has the coordinate $x_{q}=x_{0}+\alpha r_{01}+\alpha \beta r_{12}, \alpha \in[0,1], \beta \in[0,1]$, as displayed in Fig. S5. Using $\alpha, \beta$ as the surface coordinates, a metric tensor can be obtained naturally from the embedding in $\mathbb{R}^{3}$. In the matrix form, the metric tensor writes

$$
\left(g_{\mu \nu}\right)=\left(\begin{array}{cc}
\left\|r_{01}+\beta r_{12}\right\|^{2} & \left\langle\alpha r_{12}, r_{01}+\beta r_{12}\right\rangle \\
\left\langle\alpha r_{12}, r_{01}+\beta r_{12}\right\rangle & \left\|\alpha r_{12}\right\|^{2}
\end{array}\right)
$$

where each element $g_{\mu \nu}$ is the inner product between tangent vectors $\partial_{\mu}$ and $\partial_{\nu}$, where $\mu, \nu$ can be $\alpha$ or $\beta$. Let $g$ be the determinant of the metric tensor. Then

$$
\begin{aligned}
g & =\left\|r_{01}+\beta r_{12}\right\|^{2}\left\|\alpha r_{12}\right\|^{2}-\left\langle\alpha r_{12}, r_{01}+\beta r_{12}\right\rangle^{2} \\
& =\left(\alpha\left\|r_{01} \times r_{12}\right\|\right)^{2}
\end{aligned}
$$

where $\left\|r_{01} \times r_{12}\right\|$ is simply twice the area of the triangle $A(t)$.

Suppose we have a filament tip $p$ at position $x_{p}$, and let $r_{p 0}=x_{0}-x_{p}$, then the distance between $p$ and $q$ is

$$
d=\left\|\alpha r_{01}+\alpha \beta r_{12}+r_{p 0}\right\|
$$

The interaction energy can be written as

$$
\hat{E}_{\text {excl }}(t, p)=k_{\text {excl }} \int_{t} d S \frac{1}{d^{4}}
$$

where $k_{\text {excl }}$ is the strength of the interaction. The area element is the volume form on the surface, and can be expressed as

$$
\begin{aligned}
d S & =\sqrt{g} d \alpha \wedge d \beta \\
& =\alpha\left\|r_{01} \times r_{12}\right\| d \alpha \wedge d \beta
\end{aligned}
$$

Now Eq. 13 can be written as

$$
\hat{E}_{\text {excl }}=k_{\text {excl }}\left\|r_{01} \times r_{12}\right\| \underbrace{\int_{0}^{1} \alpha d \alpha \int_{0}^{1} d \beta\left\|\alpha r_{01}+\alpha \beta r_{12}+r_{p 0}\right\|^{-4}}_{I}
$$




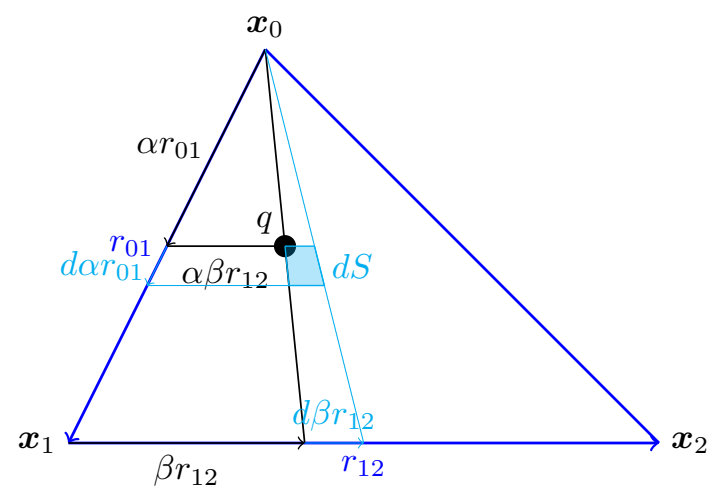

Figure S5: The area element on a triangle facet.

Then by letting

$$
\begin{aligned}
a & =\left\|r_{01}\right\|^{2} \\
b & =\left\|r_{12}\right\|^{2} \\
c & =\left\|r_{p 0}\right\|^{2} \\
e & =\left\langle r_{01}, r_{p 0}\right\rangle \\
f & =\left\langle r_{12}, r_{p 0}\right\rangle \\
g & =\left\langle r_{01}, r_{12}\right\rangle
\end{aligned}
$$

one can get

$$
I=\int_{0}^{1} \alpha d \alpha \int_{0}^{1} d \beta\left(\alpha^{2} a^{2}+\alpha^{2} \beta^{2} b^{2}+c^{2}+2 e+2 \alpha \beta f+2 \alpha^{2} \beta g\right)^{-2}
$$

we are able to evaluate the integral analytically with

$$
\begin{aligned}
& I=\left(-\frac{(a f-e g) \arctan \frac{e}{\sqrt{a c-e^{2}}}}{\sqrt{a c-e^{2}}}\right. \\
&+\frac{(a f-e g) \arctan \frac{a+e}{\sqrt{a c-e^{2}}}}{\sqrt{a c-e^{2}}} \\
&+\frac{(a f+f g-b e-e g) \arctan \frac{e+f}{\sqrt{a c-e^{2}-2 e f-f^{2}+b c+2 c g}}}{\sqrt{a c+b c-e^{2}-2 e f-f^{2}+2 c g}} \\
&+\frac{(b e-a f+(e-f) g) \arctan \frac{a+b+e+f+2 g}{\sqrt{a c-e^{2}-2 e f-f^{2}+b c+2 c g}}}{\sqrt{a c+b c-e^{2}-2 e f-f^{2}+2 c g}} \\
&+\frac{(a b+b e-g(f+g)) \arctan \frac{f+g}{\sqrt{b(a+c)+2 b e-(f+g)^{2}}}}{\sqrt{b(a+c)+2 b e-(f+g)^{2}}} \\
&\left.-\frac{(a b+b e-g(f+g)) \arctan \frac{b+f+g}{\sqrt{b(a+c)+2 b e-(f+g)^{2}}}}{\sqrt{b(a+c)+2 b e-(f+g)^{2}}}\right) \\
& 2\left(b e^{2}+a\left(-b c+f^{2}\right)+g(-2 e f+c g)\right)
\end{aligned}
$$

Then the $j$-th component of the force on vertex $v_{i}$ is $f_{i j}=-\partial \hat{E}_{\text {excl }} / \partial x^{j}\left(v_{i}\right)$, and can be derived using the chain rule. 


\subsection{Geometric interpretation of Eq. 18}

Let $\iota$ be the denominator in Eq. 18, and then

$$
\begin{aligned}
\iota= & 2\left(b e^{2}+a\left(-b c+f^{2}\right)+g(-2 e f+c g)\right) \\
= & 2\left[\left\|r_{12}\right\|^{2}\left\langle r_{01}, r_{p 0}\right\rangle^{2}-\left\|r_{01}\right\|^{2}\left(\left\|r_{12}\right\|^{2}\left\|r_{p 0}\right\|^{2}-\left\langle r_{12}, r_{p 0}\right\rangle^{2}\right)\right. \\
& \left.-2\left\langle r_{01}, r_{12}\right\rangle\left\langle r_{01}, r_{p 0}\right\rangle\left\langle r_{12}, r_{p 0}\right\rangle+\left\|r_{p 0}\right\|^{2}\left\langle r_{01}, r_{12}\right\rangle^{2}\right] \\
= & -2\left\langle\left(r_{01} \times r_{12}\right), r_{p 0}\right\rangle^{2}
\end{aligned}
$$

which is -72 times the square of volume $V$ of the tetrahedron formed by $x_{0}, x_{1}, x_{2}$ and $x_{p}$. Note that $\iota=0$ when the four points lie in the same plane, but when the triangle is not degenerate, $I$ is only infinity when the point $x_{p}$ lies inside the triangle $t$.

Given the substitutions in Eq. 16, we can define some other values expressed using these substitutions.

$$
\begin{aligned}
h & =\left\|r_{02}\right\|^{2}=a+b+2 g \\
l & =\left\|r_{p 1}\right\|^{2}=a+c+2 e \\
m & =\left\|r_{p 2}\right\|^{2}=a+b+c+2(e+f+g) \\
o & =\left\langle r_{12}, r_{p 1}\right\rangle=f+g \\
p & =\left\langle r_{01}, r_{02}\right\rangle=a+g \\
q & =\left\langle r_{01}, r_{p 1}\right\rangle=a+e \\
r & =\left\langle r_{02}, r_{p 0}\right\rangle=e+f
\end{aligned}
$$

The terms in the integral that show up in the square roots can also have geometric interpretation

$$
\begin{aligned}
\delta_{2} & =a c-e^{2} \\
& =\left\|r_{01}\right\|^{2}\left\|r_{p 0}\right\|^{2}-\left\langle r_{01}, r_{p 0}\right\rangle^{2} \\
& =\left\|r_{01} \times r_{p 0}\right\|^{2} \\
\delta_{1}= & a c+b c-e^{2}-2 e f-f^{2}+2 c g \\
= & c(a+b+2 g)-(e+f)^{2} \\
= & c h-r^{2} \\
= & \left\|r_{02} \times r_{p 0}\right\|^{2} \\
\delta_{0} & =b(a+c)+2 b e-(f+g)^{2} \\
& =b l-o^{2} \\
& =\left\|r_{12} \times r_{p 1}\right\|^{2}
\end{aligned}
$$

And then the coefficients before each arctan can be further written as

$$
\begin{aligned}
\epsilon_{2} & =-(a f-e g) / \sqrt{\delta_{2}} \\
& =\left\|r_{01} \times r_{12}\right\| \frac{-\left\|r_{01}\right\|^{2}\left\langle r_{12}, r_{p 0}\right\rangle+\left\langle r_{01}, r_{p 0}\right\rangle\left\langle r_{01}, r_{12}\right\rangle}{\left\|r_{01} \times r_{p 0}\right\|\left\|r_{01} \times r_{12}\right\|} \\
& =2 A_{t} \cos \varphi_{2}
\end{aligned}
$$

where $\varphi_{i}$ is the dihedral angle between $t$ and the triangle formed by $x_{p}, x_{i+1}$ and $x_{i+2}$ (where the plus in the subscripts takes the modulo of 3 ). Likewise,

$$
\begin{aligned}
\epsilon_{1} & =(a f+f g-b e-e g) / \sqrt{\delta_{1}} \\
& =(-e(a+b+2 g)+(a+g)(e+f)) / \sqrt{\delta_{1}} \\
& =(-e h+p r) / \sqrt{\delta_{1}} \\
& =2 A(t) \cos \varphi_{1}
\end{aligned}
$$




$$
\begin{aligned}
\epsilon_{0} & =(a b+b e-g(f+g)) / \sqrt{\delta_{0}} \\
& =(b q-g o) / \sqrt{\delta_{0}} \\
& =2 A(t) \cos \varphi_{0}
\end{aligned}
$$

Finally, the arctan terms can have their interpretation as well, using the fact that $\arctan (1 / \tan (x))=$ $\frac{\pi}{2}-x$ when $x \in[0, \pi]$.

$$
\begin{aligned}
\zeta_{2} & =\arctan \frac{e}{\sqrt{\delta_{2}}}-\arctan \frac{a+e}{\sqrt{\delta_{2}}} \\
& =\arctan \cot (\pi-\angle p 01)-\arctan \cot \angle p 10 \\
& =\angle p 01-\frac{\pi}{2}-\left(\frac{\pi}{2}-\angle p 10\right) \\
& =-\angle 0 p 1 \\
\zeta_{1} & =\arctan \frac{e+f}{\sqrt{\delta_{1}}}-\arctan \frac{a+b+e+f+2 g}{\sqrt{\delta_{1}}} \\
& =\arctan \cot (\pi-\angle p 02)-\arctan \cot \angle p 20 \\
& =\angle p 02-\frac{\pi}{2}-\left(\frac{\pi}{2}-\angle p 20\right) \\
& =-\angle 2 p 0 \\
\zeta_{0} & =\arctan \frac{f+g}{\sqrt{\delta_{0}}}-\arctan \frac{b+f+g}{\sqrt{\delta_{0}}} \\
& =\arctan \cot (\pi-\angle p 12)-\arctan \cot \angle p 21 \\
& =\angle p 12-\frac{\pi}{2}-\left(\frac{\pi}{2}-\angle p 21\right) \\
& =-\angle 1 p 2
\end{aligned}
$$

So Eq. 18 can be rewritten as

$$
\begin{aligned}
I & =\left(\sum_{i=0}^{2} \epsilon_{i} \zeta_{i}\right) /\left(-72 V^{2}\right) \\
& =\frac{A(t)}{36 V^{2}}\left(\cos \varphi_{0} \cdot \angle 1 p 2+\cos \varphi_{1} \cdot \angle 2 p 0+\cos \varphi_{2} \cdot \angle 0 p 1\right)
\end{aligned}
$$

And Eq. 13 would be

$$
\begin{aligned}
\hat{E}_{\mathrm{excl}} & =\frac{k_{\mathrm{excl}} A(t)^{2}}{18 V^{2}}\left(\cos \varphi_{0} \cdot \angle 1 p 2+\cos \varphi_{1} \cdot \angle 2 p 0+\cos \varphi_{2} \cdot \angle 0 p 1\right) \\
& =\frac{\pi k_{\mathrm{excl}}}{d_{\perp}^{2}} \eta(t, p)
\end{aligned}
$$

where $d_{\perp}$ is the distance between point $p$ and the plane defined by triangle $t$, and $\eta(t, p)=$ $\left(\cos \varphi_{0} \cdot \angle 1 p 2+\cos \varphi_{1} \cdot \angle 2 p 0+\cos \varphi_{2} \cdot \angle 0 p 1\right) /(2 \pi)$ can be considered as the "contribution factor" of the triangle inside the whole plane corresponding to the case $n=4$. When the triangle grows infinitely large to span the whole plane, $\cos \varphi_{i}=1$, and $\angle 1 p 2+\angle 2 p 0+\angle 0 p 1=2 \pi$, so in this case the integral becomes $E_{\text {excl }}=\pi k_{\text {excl }} / d_{\perp}^{2}$, which is exactly Eq. 13 integrated over the whole plane.

\section{Performance of bundle protrusion simulations}

The membrane model introduces a lot of new elements and new interactions into the original MEDYAN, bringing new computational challenges. Every simulation in this work was run on a single Intel Ivy Bridge E5-2680v2 CPU core running at $2.80 \mathrm{GHz}$. Below, we show performance data for 
some of the bundle protrusion simulations, because these simulations involve highly fine-grained membrane surface (as a result of remeshing with very high local curvature along the tube) and close interactions between the membrane and the actin filaments.

Three trajectories of perpendicular bundle protrusion are analyzed, with the same initial G-actin concentration $0.055 \mathrm{~mol} / \mathrm{m}^{3}$, but different number of filaments in the bundles. All these bundles successfully form finger-like protrusions. The following tables (Table S4 S5 and S6 show the wall time taken for various processes in a $0.2 \mathrm{~s}$ simulation time window at different stages of filopodial growth, with number of filaments in the bundle, $N$, being 7, 10 and 14, respectively. The first column in each table indicates the End of each Simulation Time Window (ESTW). For example, if the ESTW is $2.0 \mathrm{~s}$, the actual simulation time window is $1.8-2.0 \mathrm{~s}$. The number of membrane triangles, filament beads, ${ }^{1}$ and the height of the tube are taken at each ESTW.

Table S4: Time consumption of $0.2 \mathrm{~s}$ simulation time window when $N=7$. Simulating $16.1 \mathrm{~s}$ in total took $1 \mathrm{~d} 6 \mathrm{~h} 22 \mathrm{~min}$.

\begin{tabular}{lrrrrrrrrr}
\hline & \multicolumn{3}{c}{ System states } & & \multicolumn{5}{c}{ MEDYAN time consumption } \\
\cline { 2 - 3 } ESTW & \# triangles & \# beads & Height & & Chemistry & Mechanics & Remesh & Neighbor list & Total \\
\hline $4.0 \mathrm{~s}$ & 2700 & 104 & $835 \mathrm{~nm}$ & & $1.21 \mathrm{~s}$ & $541 \mathrm{~s}$ & $8.01 \mathrm{~s}$ & $3.96 \mathrm{~s}$ & $555 \mathrm{~s}$ \\
$8.0 \mathrm{~s}$ & 4492 & 171 & $1535 \mathrm{~nm}$ & $2.13 \mathrm{~s}$ & $1135 \mathrm{~s}$ & $14.5 \mathrm{~s}$ & $11.1 \mathrm{~s}$ & $1164 \mathrm{~s}$ \\
$12.0 \mathrm{~s}$ & 6352 & 239 & $2196 \mathrm{~nm}$ & $3.06 \mathrm{~s}$ & $2245 \mathrm{~s}$ & $20.9 \mathrm{~s}$ & $22.4 \mathrm{~s}$ & $2292 \mathrm{~s}$ \\
$16.0 \mathrm{~s}$ & 8206 & 304 & $2876 \mathrm{~nm}$ & & $5.64 \mathrm{~s}$ & $4006 \mathrm{~s}$ & $49.5 \mathrm{~s}$ & $39.8 \mathrm{~s}$ & $4102 \mathrm{~s}$ \\
\hline
\end{tabular}

Table S5: Time consumption of $0.2 \mathrm{~s}$ simulation time window when $N=10$. Simulating $10.4 \mathrm{~s}$ in total took $3 \mathrm{~d}$.

\begin{tabular}{|c|c|c|c|c|c|c|c|c|}
\hline \multirow[b]{2}{*}{ ESTW } & \multicolumn{3}{|c|}{ System states } & \multicolumn{5}{|c|}{ MEDYAN time consumption } \\
\hline & \# triangles & \# beads & Height & Chemistry & Mechanics & Remesh & Neighbor list & Total \\
\hline $2.0 \mathrm{~s}$ & 2288 & 120 & $647 \mathrm{~nm}$ & $3.21 \mathrm{~s}$ & $1993 \mathrm{~s}$ & $11.0 \mathrm{~s}$ & $5.90 \mathrm{~s}$ & $2013 \mathrm{~s}$ \\
\hline $4.0 \mathrm{~s}$ & 3612 & 170 & $1198 \mathrm{~nm}$ & $2.88 \mathrm{~s}$ & $3899 \mathrm{~s}$ & $16.7 \mathrm{~s}$ & $12.8 \mathrm{~s}$ & $3932 \mathrm{~s}$ \\
\hline $6.0 \mathrm{~s}$ & 4998 & 220 & $1738 \mathrm{~nm}$ & $4.04 \mathrm{~s}$ & $5933 \mathrm{~s}$ & $24.4 \mathrm{~s}$ & $24.8 \mathrm{~s}$ & $5988 \mathrm{~s}$ \\
\hline $8.0 \mathrm{~s}$ & 6396 & 273 & $2299 \mathrm{~nm}$ & $4.24 \mathrm{~s}$ & $5744 \mathrm{~s}$ & $26.6 \mathrm{~s}$ & $33.1 \mathrm{~s}$ & $5809 \mathrm{~s}$ \\
\hline $10.0 \mathrm{~s}$ & 7810 & 327 & $2842 \mathrm{~nm}$ & $7.89 \mathrm{~s}$ & $8873 \mathrm{~s}$ & $33.6 \mathrm{~s}$ & $50.3 \mathrm{~s}$ & $8967 \mathrm{~s}$ \\
\hline
\end{tabular}

Table S6: Time consumption of $0.2 \mathrm{~s}$ simulation time window when $N=14$. Simulating $4.01 \mathrm{~s}$ in total took $3 \mathrm{~d}$.

\begin{tabular}{|c|c|c|c|c|c|c|c|c|}
\hline \multirow[b]{2}{*}{ ESTW } & \multicolumn{3}{|c|}{ System states } & \multicolumn{5}{|c|}{ MEDYAN time consumption } \\
\hline & \# triangles & \# beads & Height & Chemistry & Mechanics & Remesh & Neighbor list & Total \\
\hline $0.8 \mathrm{~s}$ & 1700 & 136 & $407 \mathrm{~nm}$ & $4.57 \mathrm{~s}$ & $3680 \mathrm{~s}$ & $14.6 \mathrm{~s}$ & $7.34 \mathrm{~s}$ & $3708 \mathrm{~s}$ \\
\hline $1.6 \mathrm{~s}$ & 2506 & 176 & $766 \mathrm{~nm}$ & $6.66 \mathrm{~s}$ & $9177 \mathrm{~s}$ & $21.9 \mathrm{~s}$ & $15.6 \mathrm{~s}$ & $9222 \mathrm{~s}$ \\
\hline $2.4 \mathrm{~s}$ & 3266 & 215 & $1099 \mathrm{~nm}$ & $8.68 \mathrm{~s}$ & $13404 \mathrm{~s}$ & $28.4 \mathrm{~s}$ & $24.3 \mathrm{~s}$ & $13467 \mathrm{~s}$ \\
\hline $3.2 \mathrm{~s}$ & 4168 & 254 & $1446 \mathrm{~nm}$ & $10.4 \mathrm{~s}$ & $20443 \mathrm{~s}$ & $38.0 \mathrm{~s}$ & $40.3 \mathrm{~s}$ & $20534 \mathrm{~s}$ \\
\hline $4.0 \mathrm{~s}$ & 4990 & 293 & $1776 \mathrm{~nm}$ & $7.26 \mathrm{~s}$ & $21906 \mathrm{~s}$ & $43.2 \mathrm{~s}$ & $54.1 \mathrm{~s}$ & $22013 \mathrm{~s}$ \\
\hline
\end{tabular}

From the tables, it is evident that, in bundle protrusion simulations, the mechanical energy minimization takes the majority of the entire time consumption. As the protrusion grows, the number of beads required to represent the actin filaments increases, and the number of triangles in the membrane mesh also increases because the area of the high-curvature tube increases. These, in turn, increase the number of pairwise interactions between the membrane triangles and the filament beads multiplicatively, leading to performance degradation in force relaxation computations with long tubes. However, this performance impact will be much less prominent in systems where the 
membrane is not closely wrapping the actin filaments because of fewer short-range pairwise repulsive interactions between the membrane triangles and the filament beads. Moreover, the adaptive remeshing algorithm will produce larger triangles on relatively flat regions on the membrane, thus reducing the mesh element density and saving computation time. Developing parallel algorithms utilizing GPUs or multicore CPUs to boost the performance of membrane mechanical energy computations is an important future direction.

\section{References}

[1] Popov, K.; Komianos, J.; Papoian, G. A. MEDYAN: mechanochemical simulations of contraction and polarity alignment in actomyosin networks. PLoS Comput. Biol. 2016, 12, e1004877.

[2] Levine, Z. A.; Venable, R. M.; Watson, M. C.; Lerner, M. G.; Shea, J.-E.; Pastor, R. W.; Brown, F. L. Determination of biomembrane bending moduli in fully atomistic simulations. $J$. Am. Chem. Soc. 2014, 136, 13582-13585.

[3] Helfrich, W. Elastic properties of lipid bilayers: theory and possible experiments. Zeitschrift für Naturforschung C 1973, 28, 693-703.

[4] Safran, S. A. Statistical Thermodynamics of Surfaces, Interfaces, and Membranes; CRC Press, 2018; Vol. 90.

[5] Seung, H.; Nelson, D. R. Defects in flexible membranes with crystalline order. Phys. Rev. A 1988, 38, 1005-1018.

[6] Sullivan, J. M. Discrete Differential Geometry; Birkhäuser Basel, 2008; pp 175-188.

[7] Boal, D. H.; Rao, M. Topology changes in fluid membranes. Phys. Rev. A 1992, 46, 3037-3045.

[8] Kantor, Y.; Nelson, D. R. Phase transitions in flexible polymeric surfaces. Phys. Rev. A 1987, 36, 4020-4032.

[9] Li, J.; Dao, M.; Lim, C.; Suresh, S. Spectrin-level modeling of the cytoskeleton and optical tweezers stretching of the erythrocyte. Biophys. J. 2005, 88, 3707-3719.

[10] Gompper, G.; Kroll, D. Random surface discretizations and the renormalization of the bending rigidity. J. Phys. I France 1996, 6, 1305-1320.

[11] Brakke, K. A. The surface evolver. Exp. Math. 1992, 1, 141-165.

[12] Brakke, K. A. Surface evolver manual. Mathematics Department, Susquehanna Univerisity, Selinsgrove, PA 1994, 17870, 20.

[13] Meyer, M.; Desbrun, M.; Schröder, P.; Barr, A. H. Mathematics and Visualization; Springer Berlin Heidelberg, 2003; pp 35-57.

[14] Guckenberger, A.; Schraml, M. P.; Chen, P. G.; Leonetti, M.; Gekle, S. On the bending algorithms for soft objects in flows. Comput. Phys. Commun. 2016, 207, 1-23.

[15] Guckenberger, A.; Gekle, S. Theory and algorithms to compute Helfrich bending forces: A review. J. Phys.: Condens. Matter 2017, 29, 203001.

[16] Le, D.-V. Subdivision elements for large deformation of liquid capsules enclosed by thin shells. Comput. Method. Appl. M. 2010, 199, 2622-2632.

[17] Doi, A.; Koide, A. An efficient method of triangulating equi-valued surfaces by using tetrahedral cells. Ieice T. Inf. Syst. 1991, 74, 214-224. 